\title{
Live unrelated donor renal transplantation in children-criteria of acceptability
}

\author{
Richard N. Fine
}

Received: 1 December 2008 / Accepted: 4 December 2008/Published online: 25 March 2009

(C) IPNA 2009

Sirs,

I read with great interest the manuscript by Gheith et al. describing the outcome of live unrelated donor (LURD) pediatric kidney transplantation in Egypt [1].

Certainly, there is a shortage of donor organs for solid organ transplantation worldwide, although the revised criteria for distribution of deceased donors' kidneys to pediatric patients by the United Network for Organ Sharing (UNOS) in the United States of America has minimized the waiting time for children. Increasing the availability of organs for kidney transplantation needs to be a priority, so that morbidity and the mortality rate in end-stage renal disease (ESRD) may be reduced worldwide.

As President of the American Society of Transplantation (AST), I indicated in my 2006 presidential address that I felt that the transplantation community needed to rethink its opposition to incentivization as a method for increasing the availability of organ donors. However, the methodology described in the report by Gheith et al. [1] is suboptimal in my opinion, for the following reasons:

1. The requirement that all potential family donors be interrogated prior to the potential for accessing a LURD gives the appearance of coercion.

R. N. Fine $(\bowtie)$

School of Medicine, Stony Brook University Medical Center, Stony Brook, NY 11794-843, USA

e-mail: richard.fine@stonybrook.edu
2. There is no description of the methodology by which potential LURDs were identified-individual recipient solicitation or local/national advertising campaign - and how the LURD organs were distributed. The latter is exceedingly important, because, if specific criteria are developed for distribution not on the basis of need, the system lacks equity. A central clearing house for organ distribution would be optimal.

3. The lack of a defined system of incentivization facilitates a system that permits individual resources to dictate acquisition or distribution and, therefore, lacks equity.

4. There is no provision for the LURD to receive free lifetime medical care and a system to examine periodically the health status of the donor to identify and potentially treat any adverse consequences from donation.

Perhaps the authors would like to address these concerns.

\section{References}

1. Gheith O, Sabry A, El-Baset SA, Hassan N, Sheashaa H, Bahgat S, El-Shahawy El-M (2008) Study of the effect of donor source on graft and patient survival in pediatric renal transplant recipients. Pediatr Nephrol 23:2075-2079 